\title{
On Certain Trigonometrical Expansions
}

\section{H. A. LAUWERIER}

\author{
Communicated by A. ERDÉLyI
}

1. Introduction. The work described in this paper arises from a study of the long waves in the North Sea generated by a wind field.

In this context there occurs the problem of ${ }^{1.2}$ how to determine a solution of the equation of HeLmHoLtz

$$
\frac{\partial^{2} F}{\partial x^{2}}+\frac{\partial^{2} F}{\partial y^{2}}-q^{2} F=0,
$$

in the region $0<x<\pi, y>0$, which vanishes for $y \rightarrow \infty$, and which satisfies the boundary conditions

$$
\begin{gathered}
F=0 \text { at } x=0 \text { and } x=\pi \\
\cos \mu \pi \frac{\partial F}{\partial y}-\sin \mu \pi \frac{\partial F}{\partial x}+f(x)=0 \text { at } y=0 .
\end{gathered}
$$

It will be assumed that $q$ and $\mu$ are given real constants with $q \geqq 0$ and $0 \leqq \mu \leqq \frac{1}{2}$, and that $f(x)$ is a given real function. In order to simplify the discussion we shall assume that $f(x)$ is an analytic function. In many cases, however, a much less stringent condition may be sufficient.

The condition (1.3) means that at $y=0$ the solution $F(x, y)$ has a prescribed directional derivative, the direction of which makes a positive angle $\mu \pi$ with the normal. Physically $\operatorname{tg} \mu \pi$ is proportional to the coefficient of CoRIoLIs, i.e. the angular velocity of the earth.

The solution $F(x, y)$ may be represented as follows

$$
F(x, y)=\sum_{1}^{\infty} b_{k} \frac{\sin k x}{k} e^{-y \sqrt{k^{2}+q^{2}}} .
$$

The coefficients $b_{k}$ can be determined by means of the boundary condition (1.3) at $y=0$ which gives

$$
f(x)=\sum_{1}^{\infty} b_{k}\left\{\sqrt{1+\frac{q^{2}}{k^{2}}} \cos \mu \pi \sin k x+\sin \mu \pi \cos k x\right\} .
$$

${ }^{1}$ Cf. G. I. Taylor, Proc. Lond. Math. Soc. (2) XX 148 (1920).

${ }^{2} C f$. H. Lamb, Hydrodynamics, $6^{\text {th }}$ ed., art. 209 (1932). 
(a) For $q=0, \mu=0$ this relation reduces to a sine expansion

$$
f(x)=\sum_{1}^{\infty} b_{k} \sin k x .
$$

In this case the coefficients $b_{k}$ may be determined in the familiar way and they are of harmonic order $b_{k}=O\left(k^{-1}\right)$.

(b) For $q=0, \mu=\frac{1}{2} \pi(1.5)$ reduces to a cosine expansion

$$
f(x)=\sum_{1}^{\infty} b_{k} \cos k x
$$

In this case no convergent expansion exists unless $\int_{0}^{\pi} f(t) d t=0$.

(c) For $q=0,0<\mu<\frac{1}{2}(1.5)$ reduces to

$$
f(x)=\sum_{1}^{\infty} b_{k} \sin (k x+\mu \pi) \text {. }
$$

This expansion will be the subject of the first part of this paper. The results are as follows: The expansion (1.8) is unique and $b_{k}$ is of subharmonic order $b_{k}=O\left(k^{-1+2 \mu}\right)$.

In particular, we have the special case

$$
1=\sum_{1}^{\infty}(-1)^{k-1} e_{k}(2 \mu) \sin (k x+\mu \pi), \quad 0<x<\pi,
$$

where $e_{k}(\alpha)$ is defined by the generating function

$$
\left(\frac{1+s}{1-s}\right)^{\alpha}=\sum_{0}^{\infty} e_{k}(\alpha) s^{k}, \quad|s|<1 \text {. }
$$

We have

$$
e_{0}=1, \quad e_{1}=2 \alpha, \quad e_{2}=2 \alpha^{2}, \cdots
$$

and for arbitrary index $k \geqq 1$

$$
e_{k}(\alpha)=(-1)^{k-1} 2 \alpha F(1-k, 1+\alpha ; 2 ; 2) .
$$

The coefficients $b_{k}$ of (1.8) may be expressed by means of

$$
b_{k}=\frac{1}{\pi} \int_{0}^{\pi} k_{k}(t) f(t) d t
$$

where $k_{m}(x)$ is the $m^{\text {th }}$ orthogonal function with respect to the set $\sin (n x+\mu \pi)$. These functions may be determined by the generating function

$$
\sum_{1}^{\infty} k_{m}(x) s^{m}=\left(\frac{1+s}{1-s} \operatorname{tg} \frac{x}{2}\right)^{-2 \mu} \sum_{1}^{\infty} 2 \sin k x s^{k} .
$$

The functions $k_{m}(x)$ are uniformly bounded. For all $m \geqq 1$ and all $x$ in $(0, \pi)$ we have

$$
\left|k_{m}(x)\right| \leqq 2+4 \sin \mu \pi
$$


We note that the function $\left(\operatorname{tg} \frac{1}{2} x\right)^{-2 \mu}$ is orthogonal with respect to all functions of the set $\sin (n x+\mu \pi)$, viz.

$$
\frac{1}{\pi} \int_{0}^{\pi}\left(\operatorname{tg} \frac{t}{2}\right)^{-2 \mu} \sin (n t+\mu \pi) d t=\delta_{0 n}
$$

for $n=0,1,2, \cdots$. If for the function $f(x)$ of (1.8) the following condition is fulfilled:

$$
\frac{1}{\pi} \int_{0}^{\pi}\left(\operatorname{tg} \frac{t}{2}\right)^{-2 \mu} f(t) d t=0
$$

then the coefficients $b_{k}$ are of hyperharmonic order $b_{k}=O\left(k^{-1-2 \mu}\right)$.

(d) We shall consider the slightly more general case

$$
f(x)=\sum_{1}^{\infty} b_{k}\left(\sin k x+\theta_{k} \cos k x\right), \quad 0<x<\pi,
$$

where $\theta_{k}=\theta+O\left(\theta^{-c}\right), c>1$, for $k \rightarrow \infty$. In the case given by (1.5) we have

$$
\theta_{k}=\left(1+\frac{q^{2}}{k^{2}}\right)^{-\frac{1}{2}} \operatorname{tg} \mu \pi, \quad \theta=\operatorname{tg} \mu \pi .
$$

Relatively little seems to be known concerning expansions of this kind. We quote the following result due to PALEY \& WIENER ${ }^{3}$ If $\left|\theta_{k}\right| \leqq c \leqq 1$ and if $f(x) \& L^{2}$ a unique expansion of the kind (1.15) exists with $\sum_{1}^{\infty} b_{k}^{2}<\infty$.

In section 5 it will be shown that the expansion problem (1.15) may be reduced to either an infinite system of linear equations or an integral equation of the Fredholm type.

The results are as follows: For $\theta>0$ a convergent expansion of the kind (1.15) exists and is unique. The coefficients $b_{k}$ are of subharmonic order

$$
b_{k}=\frac{(-1)^{k} \beta}{k^{1-2 \mu}}+O\left(k^{-1-2 \mu}\right), \quad k \rightarrow \infty,
$$

where $\beta$ does not depend on $k$, and where $\mu=(1 / \pi) \operatorname{arctg} \theta$.

In a way similar to the preceding case the coefficient $b_{k}$ may be written in the form

$$
b_{k}=\frac{1}{\pi} \int_{0}^{\pi} m_{k}(t) f(t) d t
$$

where the functions $m_{k}(x)$ are bounded in $(0, \pi)$ and orthogonal with respect to the set $\sin k x+\theta_{k} \cos k x$.

There exists a function $l_{0}(x)$ for which

$$
\frac{1}{\pi} \int_{0}^{\pi} l_{0}(t)\left(\sin n t+\theta_{n} \cos n t\right) d t=\delta_{0 n}
$$

\footnotetext{
${ }^{3}$ Fourier transforms in the complex plane, Am. Math. Soc., New York, p. 100 (1943).
} 
This function is bounded in $(0, \pi)$ with the exception of $x=0$ where, however,

$$
\left(\operatorname{tg} \frac{x}{2}\right)^{-1+2 \mu} l_{0}(x)
$$

has a finite limit.

Returning to the boundary value problem considered at the beginning we may conclude that a unique solution $F(x, y)$ exists which is continuous and bounded throughout the semi-infinite strip $0 \leqq x \leqq \pi, 0 \leqq y \leqq \infty$. The behaviour of $F(x, y)$ at the corners $x=0$ and $x=\pi, y=0$ is determined by

$x \rightarrow 0, \quad y \rightarrow 0, \quad F(x, y)=c_{1}+c_{2} r^{1+2 \mu} \sin (1+2 \mu)\left(\frac{\pi}{2}-\varphi\right)+$ higher terms $x \rightarrow \pi, \quad y \rightarrow 0, \quad F(x, y)=c_{3}+c_{4} r^{1-2 \mu} \sin (1-2 \mu)\left(\frac{\pi}{2}-\varphi\right)+$ higher terms where $(r, \varphi)$ are local polar coordinates and where $c_{1}, c_{2}, c_{3}, c_{4}$ are constants.

2. We shall consider at first the expansion of $f(x)$ into a trigonometrical series with a constant term

$$
f(x)=\sum_{0}^{\infty} a_{k} \sin (k x+\mu \pi), \quad 0<x<\pi,
$$

with $0<\mu<\frac{1}{2}$. The discussion of this expansion is somewhat simpler than that of the expansion (1.8). We shall require that

$$
\sum_{0}^{\infty}\left|a_{k}\right|<\infty \text {. }
$$

For the present purposes it is sufficient that $f(x)$ satisfies a Hölder condition of the following kind:

$$
\left|f\left(x_{1}\right)-f\left(x_{2}\right)\right|<c\left|x_{1}-x_{2}\right|^{-\gamma}
$$

for any pair $x_{1}, x_{2}$ from the closed interval $(0, \pi)$ and where $c$ and $\gamma$ are positive constants not depending on $x_{1}$ and $x_{2}$.

We shall show that under these assumptions the expansion (2.1) exists and is unique. Consider the analytic function

$$
\phi(z)=\sum_{0}^{\infty} a_{k} e^{k z i}
$$

where $z=x+y i, 0 \leqq x \leqq \pi, y \geqq 0$. This function satisfies the conditions

$$
\begin{aligned}
& \operatorname{Im} \phi=0 \text { at } x=0 \text { and } x=\pi, \\
& \operatorname{Im} e^{\mu \pi i} \phi=0 \text { at } y=0,
\end{aligned}
$$

$\phi$ uniformly bounded in $0 \leqq x \leqq \pi, y \geqq 0$. By means of the following conformal mapping the semi-infinite strip $0 \leqq x \leqq \pi, y \geqq 0$ is mapped upon the upper 
half plane

$$
w=-\cos z, \quad w=u+v i .
$$

By this the problem (2.5) is transformed into

$$
\begin{aligned}
\operatorname{Im} \phi & =0 & \text { at } \quad v & =0, & & |u|>1, \\
\operatorname{Im} e^{\mu \pi i} \phi & =g(u) & \text { at } \quad v & =0, & & |u|<1,
\end{aligned}
$$

$\phi$ uniformly bounded in $-\infty<u<\infty, v \geqq 0$, where $g(-\cos x) \equiv f(x)$. The problem (2.7) is a Hilbert problem for a half plane. ${ }^{4}$ Its solution may be represented as follows by a Cauchy integral

$$
\phi(w)=-\frac{1}{\pi}(w-1)^{1-\mu}(w+1)^{\mu} \int_{-1}^{1}(1-t)^{-1+\mu}(1+t)^{-\mu} \frac{g(t)}{t-w} d t .
$$

This expression has at $w= \pm 1$ the finite limit $g( \pm 1)$ cosec $\mu \pi$.

Any other solution of (2.7) differs from (2.8) by a solution of the corresponding homogeneous problem. The latter is solved by a linear combination of the functions

$$
(w+1)^{-\nu_{1}+\mu}(w-1)^{\nu_{2}-\mu}
$$

where $\nu_{1}$ and $\nu_{2}$ are integers with $\nu_{1} \geqq \nu_{2}$. Since these functions are infinite either at $w=-1$ or $w=1$ they do not comply with the last condition of (2.7). The problem (2.7) has therefore the unique solution (2.8).

From (2.8) we may derive

$$
\begin{aligned}
\sum_{1}^{\infty} a_{k} \sin k x & =\operatorname{Im} \phi(x) \\
& =(1+\cos x)\left(\operatorname{tg} \frac{x}{2}\right)^{2 \mu} \frac{1}{\pi} \int_{0}^{\pi}\left(\operatorname{tg} \frac{t}{2}\right)^{1-2 \mu} \frac{f(t)-f(x)}{\cos x-\cos t} d t .
\end{aligned}
$$

If $f(x)$ is analytic, from the right-hand side of (2.9) an asymptotic series for $a_{k}$ in negative powers of $k$ may be obtained. If $f(x)$ has a restricted number of derivatives a finite asymptotic series with a remainder is obtained. The first few terms of this asymptotic expression are determined by the behaviour of $\operatorname{Im} \phi(x)$ at $x=0$ and at $x=\pi$ only. By means of a familiar technique ${ }^{5}$ we obtain

$$
a_{k}=\frac{2^{1-2 \mu} \varphi(0)}{\Gamma(-2 \mu)} k^{-1-2 \mu}+(-1)^{k-1} \frac{2^{2 \mu-1} \varphi(\pi)}{\Gamma(2 \mu-2)} k^{-3+2 \mu}+O\left(k^{-3-2 \mu}\right),
$$

where

$$
\varphi(x)=\frac{1}{\pi} \int_{0}^{\pi}\left(\operatorname{tg} \frac{t}{2}\right)^{1-2 \mu} \frac{f(t)-f(x)}{\cos x-\cos t} d t .
$$

\footnotetext{
${ }^{4}$ Cf. Muskhelishvili, Singular integral equations, Groningen (1953), Ch. 10.

${ }^{5}$ C. G. Lekkerkerker \& A. H. M. Levelt, Asymptotic Expansion of a certain Fourier coefficient, Report ZW1957-012, Math. Centrum.
} 
We shall show that the expansion (2.1) is no longer unique if the condition (2.2) is dropped. We may, however, still require that $c_{k} \rightarrow 0$. This follows easily from the Hilbert problem considered above. To the particular solution (2.7) one may always add a constant multiple of $(w+1)^{-1+\mu}(w-1)^{-\mu}$. Since the resulting singularities of $\phi(z)$ at $z=0$ and $z=\pi$ remain integrable the coeffcient $c_{k}$ tends to zero.

An alternative, more elementary way is as follows: Consider the Taylor expansion

$$
\left(\frac{1+s}{1-s}\right)^{\alpha}=\sum_{0}^{\infty} e_{k}(\alpha) s^{k}, \quad|s|<1
$$

The first few coefficients are

$$
e_{0}=1, \quad e_{1}=2 \alpha, \quad e_{2}=2 \alpha^{2}, \quad e_{3}=\frac{4 \alpha^{3}+2 \alpha}{3} .
$$

They satisfy the recurrence relation

$$
k e_{k}=2 \alpha e_{k-1}+(k-2) e_{k-2} .
$$

Hence $k ! e_{k}$ is a polynomial in $2 \alpha$ with integer coefficients. We have, of course, for $e_{k}$ the expression

$$
e_{k}(\alpha)=\frac{1}{2 \pi i} \oint\left(\frac{1+s}{1-s}\right)^{\alpha} \frac{d s}{s^{k+1}}
$$

From this we may derive

$$
e_{k}(\alpha)=(-1)^{k-1} 2 \alpha F(1-k, 1+\alpha ; 2 ; 2), \quad k \geqq 1 .
$$

Either from (2.15) or from (2.16) the following asymptotic expansion may be obtained:

$$
e_{k}(\alpha)=\frac{2^{\alpha}}{\Gamma(\alpha) k^{1-\alpha}}+\frac{(-1)^{k} 2^{-\alpha}}{\Gamma(-\alpha) k^{1+\alpha}}+\frac{\alpha}{12} \frac{2^{\alpha}}{\Gamma(\alpha-2) k^{3-\alpha}}+O\left(k^{-3-\alpha}\right),
$$

where $0<\alpha<1$.

If in (2.13), with $-1<\alpha<1$ and $0<x<\pi, s=e^{i x}$ is substituted we obtain, separating real and imaginary parts,

$$
\begin{aligned}
& \cos \frac{\alpha \pi}{2}\left(\operatorname{tg} \frac{x}{2}\right)^{-\alpha}=\sum_{0}^{\infty} e_{k}(\alpha) \cos k x \\
& \sin \frac{\alpha \pi}{2}\left(\operatorname{tg} \frac{x}{2}\right)^{-\alpha}=\sum_{1}^{\infty} e_{k}(\alpha) \sin k x .
\end{aligned}
$$

In particular, we obtain for $\alpha=-2 \mu$

$$
0=\sum_{0}^{\infty} e_{k}(-2 \mu) \sin (k x+\mu \pi) \text {. }
$$


This is an expansion of zero into a slowly converging trigonometric series for which the condition (2.2) is violated.

We return now to the original expansion (2.1). For the coefficient $a_{0}$ we find easily by means of $a_{0}=\lim \phi(w)$ for $w \rightarrow \infty$

$$
a_{0}=\frac{1}{\pi} \int_{0}^{\pi}\left(\operatorname{tg} \frac{t}{2}\right)^{1-2 \mu} f(t) d t .
$$

By combining the expansions (2.1) and (2.20) an expansion of $f(x)$ into a trigonometric series without constant term is obtained.

$$
f(x)=\sum_{1}^{\infty} b_{k} \sin (k x+\mu \pi),
$$

where

$$
b_{k}=a_{k}-a_{0} e_{k}(-2 \mu) .
$$

From (2.11) and (2.17) we obtain the asymptotic expression

$$
b_{k}=\frac{(-1)^{k-1} 2^{2 \mu}}{\Gamma(2 \mu)} \frac{A}{k^{1-2 \mu}}+\frac{2^{-2 \mu}}{\Gamma(-2 \mu)} \frac{B}{k^{1+2 \mu}}+\frac{(-1)^{k-1} 2^{2 \mu-2}}{\Gamma(2 \mu-2)} \frac{C}{k^{3-2 \mu}}+O\left(k^{-3-2 \mu}\right),
$$

where

$$
A=a_{0}, \quad B=2 \varphi(0)-A, \quad C=2 \varphi(\pi)+\frac{2}{3} \mu A .
$$

3. The biorthogonal functions. We shall derive a set of integrable functions $h_{m}(x), m=0,1,2, \cdots$, which are orthogonal with respect to the set $\sin (n x+\mu \pi)$, $n=0,1,2, \cdots$. Thus we require that

$$
\frac{1}{\pi} \int_{0}^{\pi} h_{m}(t) \sin (n t+\mu \pi) d t=\delta_{m n}
$$

for $m, n \geqq 0$.

From (2.18) and (2.19) we obtain with $\alpha=-1+2 \mu$

$$
\begin{array}{ll}
\frac{1}{\pi} \int_{0}^{\pi}\left(\operatorname{tg} \frac{t}{2}\right)^{1-2 \mu} \cos k t d t=\frac{e_{k}(-1+2 \mu)}{2 \sin \mu \pi}, & k \geqq 1, \\
\frac{1}{\pi} \int_{0}^{\pi}\left(\operatorname{tg} \frac{t}{2}\right)^{1-2 \mu} \sin k t d t=\frac{-e_{k}(-1+2 \mu)}{2 \cos \mu \pi}, & k \geqq 1 .
\end{array}
$$

Hence it follows that

$$
\frac{1}{\pi} \int_{0}^{\pi}\left(\operatorname{tg} \frac{t}{2}\right)^{1-2 \mu} \sin (n t+\mu \pi) d t=0
$$

for $n \geqq 1$. Thus we find with the proper normalisation

$$
h_{0}(x)=\left(\operatorname{tg} \frac{x}{2}\right)^{1-2 \mu}
$$


From (3.2) and (3.3) we obtain for $m<n$

$$
\frac{1}{\pi} \int_{0}^{\pi}\left(\operatorname{tg} \frac{t}{2}\right)^{1-2 \mu} \cos m t \sin (n t+\mu \pi) d t=0
$$

so that $h_{m}(x)$ can be represented by the product of $h_{0}(x)$ and a finite cosine series. Without difficulty we obtain

$$
h_{m}(x)=\left(\operatorname{tg} \frac{x}{2}\right)^{1-2 \mu} \sum_{0}^{m} \epsilon_{j} e_{m-j}(1-2 \mu) \cos j x,
$$

where $\epsilon_{i}=1$ for $j=0$ and $\epsilon_{i}=2$ for $j \geqq 1$.

We note that the functions $h_{m}(x)$ may be derived from the generating function

$$
\sum_{0}^{\infty} h_{m}(x) s^{m}=\left(\frac{1+s}{1-s} \operatorname{tg} \frac{x}{2}\right)^{1-2 \mu} \frac{1-s^{2}}{1-2 s \cos x+s^{2}} .
$$

Finally we mention the asymptotic relation

$$
h_{m}(x)=2 \sin (m x+\mu \pi)+O\left(m^{-1-2 \mu}\right)
$$

for $0<x<1, m \rightarrow \infty$.

The coefficients $a_{k}$ of the rapidly convergent expansion

$$
f(x)=\sum_{0}^{\infty} a_{k} \sin (k x+\mu \pi)
$$

may be expressed by means of the biorthogonal functions $h_{m}(x)$ in the following way:

$$
a_{k}=\frac{1}{\pi} \int_{0}^{\pi} h_{k}(t) f(t) d t
$$

From the biorthogonal set $h_{m}(x), m=0,1,2, \cdots$, we may derive a reduced biothogonal set of functions $k_{m}(x), m=1,2, \cdots$, which are bounded in the closed interval $(0, \pi)$. We shall define

$$
k_{m}(x)=h_{m}(x)-e_{m}(-2 \mu) h_{0}(x), \quad m \geqq 1 .
$$

By means of (3.8) we obtain for $k_{m}(x)$ the generating function

$$
k(x, s) \equiv \sum_{1}^{\infty} k_{m}(x) s^{m}=\left(\frac{1+s}{1-s} \operatorname{tg} \frac{x}{2}\right)^{-2 \mu} \frac{2 s \sin x}{1-2 s \cos x+s^{2}} .
$$

This gives the following explicit representation

$$
k_{m}(x)=\left(\operatorname{tg} \frac{x}{2}\right)^{-2 \mu} \sum_{1}^{m} 2 e_{m-j}(-2 \mu) \sin j x .
$$

The coefficients $b_{k}$ of the slowly convergent expansion

$$
f(x)=\sum_{1}^{\infty} b_{k} \sin (k x+\mu \pi)
$$


may be expressed by means of the reduced biorthogonal functions. In view of (2.23) and (3.12) we have

$$
b_{k}=\frac{1}{\pi} \int_{0}^{\pi} k_{k}(t) f(t) d t .
$$

Finally we shall show that the functions $k_{m}(x)$ admit the following uniform bound:

$$
\left|k_{m}(x)\right| \leqq 2+4 \sin \mu \pi .
$$

The proof is as follows: From (3.13) we obtain

$$
k_{m}(x)=\frac{1}{2 \pi i} \oint k(x, s) s^{-m-1} d s .
$$

The function $k(x, s)$ has in the complex $s$-plane poles at $s=e^{ \pm x i}$ and branch points at $s= \pm 1$. The contribution of the poles equals $2 \sin (m x+\mu \pi)$. The contributions of the branch points are equal to two integrals which can be determined in the following way:

$k_{m}(x)=2 \sin (m x+\mu \pi)-\left(\sin \frac{x}{2}\right)^{1-2 \mu}\left(\cos \frac{x}{2}\right)^{1+2 \mu} \frac{4 \sin 2 \mu \pi}{\pi}$

$$
\cdot\left\{\alpha(\mu, x)+(-1)^{m} \alpha(-\mu, \pi-x)\right\}
$$

where

$$
\alpha(\mu, x)=\int_{1}^{\infty} \frac{(t-1)^{2 \mu}(t+1)^{2 \mu}}{t^{2}-2 t \cos x+1} \frac{d t}{t^{m}}
$$

The inequality (3.17) follows easily from the following two estimates:

$$
\begin{aligned}
\alpha(\mu, x) & \leqq 2^{-2 \mu} \int_{0}^{\infty} \frac{u^{2 \mu} d u}{u^{2}+2(1-\cos x)}=\left(\sin \frac{x}{2}\right)^{-1+2 \mu} \frac{\pi}{4 \cos \mu \pi}, \\
\alpha(-\mu, \pi-x) & \leqq 2^{2 \mu} \int_{0}^{\infty} \frac{u^{-2 \mu} d u}{u^{2}+2(1+\cos x)}=\left(\cos \frac{x}{2}\right)^{-1-2 \mu} \frac{\pi}{4 \cos \mu \pi}
\end{aligned}
$$

4. Analytic continuation. The results of the preceding two sections may easily be extended to complex values of $\mu$. Without loss of generality we may suppose that $0 \leqq \operatorname{Re} \mu \leqq \frac{1}{2}$. Since the case $0<\operatorname{Re} \mu<\frac{1}{2}$ does not lead to special considerations we restrict ourselves to the cases $\operatorname{Re} \mu=0$ and $\operatorname{Re} \mu=\frac{1}{2}$.

(a) $\operatorname{Re} \mu=\frac{1}{2}$.

We may put

$$
\left\{\begin{array}{l}
\mu=\frac{1}{2}+\frac{1}{2 \pi i} \ln \frac{\theta-1}{\theta+1}, \quad \theta>1 \\
\operatorname{tg} \mu \pi=\theta i
\end{array}\right.
$$

The rapidly convergent expansion (2.1) is possible. We have $a_{k}=O\left(k^{-2}\right)$. 
An expansion without constant term is generally not possible since $e_{k}(2 \mu)$ does not have a limit for $k \rightarrow \infty$. In this case the full set of biorthogonal functions $h_{m}(x), m=0,1,2, \cdots$, exists.

(b) $\operatorname{Re} \mu=0$.

We may put

$$
\left\{\begin{array}{l}
\mu=\frac{1}{2 \pi i} \ln \frac{1-\theta}{1+\theta}, \quad-1<\theta<1, \\
\operatorname{tg} \mu \pi=\theta i .
\end{array}\right.
$$

The slowly convergent expansion (2.22) is possible. The asymptotic behaviour of $b_{k}$ is, according to (2.24),

$$
b_{k}=\frac{(-1)^{k-1} 2^{2 \mu}}{\Gamma(2 \mu)} \frac{A}{k^{1-2 \mu}}+\frac{2^{-2 \mu}}{\Gamma(-2 \mu)} \frac{B}{k^{1+2 \mu}}+O\left(k^{-3}\right) .
$$

Hence $b_{k}$ contains two harmonic terms. Nothing regarding speed of convergence can be gained by including a single constant term in the trigonometric expansion since both $A$ and $B$ must vanish in order to get something better than harmonic convergence. The explicit expressions of $A$ and $B$ follow from (2.25). They are

$$
\begin{aligned}
& A=\frac{1}{\pi} \int_{0}^{\pi}\left(\operatorname{tg} \frac{t}{2}\right)^{1-2 \mu}\{f(t)-f(\pi)\} d t+\frac{f(\pi)}{\sin \mu \pi}, \\
& B=\frac{1}{\pi} \int_{0}^{\pi}\left(\operatorname{tg} \frac{t}{2}\right)^{-1-2 \mu}\{f(t)-f(0)\} d t-\frac{f(0)}{\sin \mu \pi} .
\end{aligned}
$$

These expressions are valid in the strip $-\frac{1}{2}<\operatorname{Re} \mu<\frac{1}{2}$. We note that for Re $\mu>0$ the expression (4.3) may be reduced to the simpler form (2.21). For $\operatorname{Re} \mu<0$ the expression (4.4) may be simplified likewise. In this case the set of biorthogonal functions $k_{m}(x), m=1,2, \cdots$, should be used since the functions $h_{m}(x)$ have a non-integrable singularity at $x=\pi$.

5. In this section we shall consider the more general expansion

$$
f(x)=\sum_{1}^{\infty} b_{k}\left(\sin k x+\theta_{k} \cos k x\right), \quad 0<x<\pi,
$$

where $\theta_{k}=\theta+O\left(\theta^{-c}\right), c>1$ for $k \rightarrow \infty$. Without loss of generality we may assume that $\theta \geqq 0$. We shall show that this expansion problem may be reduced to an integral equation. Two possible ways present themselves. If the auxiliary function

$$
s(x)=\sum_{1}^{\infty} b_{k} \sin k x
$$

is introduced the relation (5.1) may be written as

$$
s(x)+\theta S\{s(x)\}+K\{s(x)\}=f(x),
$$


where the operators $S$ and $K$ are given by

$$
\begin{aligned}
& S\{s(x)\}=\sum_{1}^{\infty} b_{k} \cos k x, \\
& K\{s(x)\}=\sum_{1}^{\infty} b_{k}\left(\theta_{k}-\theta\right) \cos k x .
\end{aligned}
$$

We have for $S$, which transforms the sine series into the conjugate cosine series,

$$
S\{s(x)\}=\frac{1}{\pi} \mathrm{P} . \mathrm{V} \cdot \int_{0}^{\pi} \frac{\sin t s(t)}{\cos x-\cos t} d t .
$$

For $K$ we have

$$
K\{s(x)\}=\frac{1}{\pi} \int_{0}^{\pi} K(x, t) s(t) d t
$$

with

$$
K(x, t)=2 \sum_{1}^{\infty}\left(\theta_{k}-\theta\right) \cos k x \sin k t .
$$

Hence (5.2) represents a singular integral equation with a singular operator $S$ and a Fredholm operator $K$. This integral equation may be solved by the method described in Muskhelishvili's book. ${ }^{6}$ This method consists in the reduction of (5.2) to an ordinary integral equation of the Fredholm type.

In the following more direct way a Fredholm equation may be derived from (5.1) immediately: If the auxiliary function

$$
\psi(x)=\sum_{1}^{\infty} b_{k}(\sin k x+\theta \cos k x)
$$

is introduced (5.1) may be written as

$$
\psi(x)+L\{\psi(x)\}=f(x)
$$

where

$$
L\{\psi(x)\}=\sum_{1}^{\infty} b_{k}\left(\theta_{k}-\theta\right) \cos k x .
$$

If we put $\theta=\operatorname{tg} \mu \pi$ with $0 \leqq \mu \leqq \frac{1}{2}$ from (5.8) the following expression for $b_{k}$ is obtained:

$$
b_{k}=\frac{\cos \mu \pi}{\pi} \int_{0}^{\pi} k_{k}(t) \psi(t) d t .
$$

Substitution into (5.10) gives

$$
L\{\psi(x)\}=\frac{1}{\pi} \int_{0}^{\pi} L(x, t) \psi(t) d t,
$$

\footnotetext{
${ }^{6}$ See footnote 4.
} 
with

$$
L(x, t)=\cos \mu \pi \sum_{1}^{\infty}\left(\theta_{k}-\theta\right) \cos k x k_{k}(t) .
$$

In view of (3.17) $L$ is a Fredholm operator with a uniformly bounded kernel. Hence (5.9) represents an ordinary Fredholm equation.

According to the familiar Fredholm theory, from (5.9) a unique and bounded solution $\psi(x)$ may be obtained. In exceptional cases, however, a certain condition should be imposed upon $f(x)$. From (2.24) we obtain for $b_{k}$ the asymptotic expression

$$
b_{k}=\frac{(-1)^{k} \beta}{k^{1-2 \mu}}+O\left(k^{-1-2 \mu}\right)
$$

where $\beta$ is a constant.

By way of illustration the following special case will be considered:

$$
\theta_{1}=\theta+\lambda, \quad \theta_{k}=\theta, \quad k \geqq 2,
$$

where $\lambda$ is variable. The Fredholm equation (5.9) reduces to

$$
\psi(x)+\cos x \frac{\lambda \cos \mu \pi}{\pi} \int_{0}^{\pi} k_{1}(t) \psi(t) d t=f(x) .
$$

Its solution is

$$
\psi(x)=f(x)+a \cos x
$$

where

$$
a\left(4 \lambda \mu^{2}+\theta\right)=-\frac{\lambda \sin \mu \pi}{\pi} \int_{0}^{\pi} k_{1}(t) f(t) d t .
$$

Thus a solution is obtained unless $4 \lambda \mu^{2}+\theta=0$. In the eigenvalue case $\lambda=$ $-\theta / 4 \mu^{2}$ the following condition must be imposed upon $f(x)$ :

$$
\int_{0}^{\pi} k_{1}(t) f(t) d t=0
$$

If to the expansion (5.1) a constant is added the rate of convergence may be improved. In a similar way as before we find that the rapidly convergent expansion

$$
f(x)=a_{0}+\sum_{1}^{\infty} a_{k}\left(\sin k x+\theta_{k} \cos k x\right), \quad 0<x<\pi,
$$

is generally possible, and that

$$
a_{k}=\frac{\alpha}{k^{1+2 \mu}}+O\left(k^{-3+2 \mu}\right)
$$

where $\alpha$ is a constant. 
Next we turn our attention to the biorthogonal set $l_{m}(x), m=0,1,2, \cdots$, with

$$
\frac{1}{\pi} \int_{0}^{\pi} l_{m}(t)\left(\sin n t+\theta_{n} \cos n t\right) d t=\delta_{m n}
$$

for $m, n \geqq 0$. The functions $l_{m}(x)$ can be obtained in the following way: Let us assume for simplicity that $\theta_{k} \neq 0$. Consider the slowly convergent expansion

$$
\cos m x=\sum_{1}^{\infty} l_{m, l}\left(\sin k x+\theta_{k}^{-1} \cos k x\right),
$$

where $m=0,1,2, \cdots$. From this expansion $l_{m}(x)$ is obtained according to

$$
l_{m}(x)=\epsilon_{m} \theta_{m}^{-1} \sum_{1}^{\infty} l_{m, k} \sin k x,
$$

where $\theta_{0}=1$. By comparing the sine and cosine coefficients of $l_{m}(x)$ the orthogonality relation (5.17) may easily be verified.

By means of the biorthogonal functions $l_{m}(x)$ the coefficients $a_{k}$ of (5.15) may be expressed by

$$
a_{k}=\frac{1}{\pi} \int_{0}^{\pi} l_{k}(t) f(t) d t, \quad k \geqq 0 .
$$

The coefficients $b_{k}$ of (5.1) may be expressed in a similar way. The reduced biorthogonal set $m_{m}(x), m=1,2, \cdots$, where $m_{m}(x)$ is the generalisation of $k_{m}(x)$, is defined by

$$
m_{m}(x)=l_{m}(x)+\lambda_{m} l_{0}(x),
$$

where

$$
1=\sum_{1}^{\infty} \lambda_{k}\left(\sin k x+\theta_{k} \cos k x\right)
$$

It is obvious that

$$
b_{k}=\frac{1}{\pi} \int_{0}^{\pi} m_{k}(t) f(t) d t, \quad k \geqq 1 .
$$

We shall, however, show that the functions $m_{m}(x)$ are bounded in the closed interval $(0, \pi)$. From (5.14) and (5.23), (5.21) we obtain

$$
b_{k}=\frac{(-1)^{k} \beta_{0}}{k^{1-2 \mu}} \int_{0}^{\pi} l_{0}(t) f(t) d t+O\left(k^{-1-2 \mu}\right),
$$

where $\beta_{0}$ does not depend on $f(x)$. The function $m_{m}(x)$ has the sine expansion

$$
m_{m}(x)=\sum_{1}^{\infty}\left(2 \theta_{m}^{-1} l_{n, k}+\lambda_{m} l_{0, k}\right) \sin k x .
$$

We shall now prove the assertion by showing that the convergence of (5.25) is of hyperharmonic order. We may apply the asymptotic expression (5.24), 
however, with $\theta_{k}$ replaced by $\theta_{k}^{-1}$. If $l_{0}^{\prime}(x)$ is orthogonal with respect to the set $\sin n t+\theta_{n}^{-1} \cos n t, n \geqq 1$, then we obtain from (5.24)

(5.26) $2 \theta_{m}^{-1} l_{n, k}+\lambda_{m} l_{0, k}=\frac{(-1)^{k} \beta_{0}^{\prime}}{k^{2 \mu}} \int_{0}^{\pi} l_{0}^{\prime}(t)\left\{2 \theta_{m}^{-1} \cos m t+\lambda_{m}\right\} d t+O\left(k^{-2+2 \mu}\right)$.

In view of the cosine expansion of $l_{0}^{\prime}(x)$,

$$
l_{0}^{\prime}(x)=\sum_{1}^{\infty} \lambda_{k} \sin k x=1-\sum_{1}^{\infty} \lambda_{k} \cos k x
$$

the integral in the right-hand side of (5.26) vanishes. Thus the convergence of (5.25) is of order $k^{-2+2 \mu}$.

The zero ${ }^{\text {th }}$ biorthogonal function $l_{0}(x)$ has a singularity similar to that of $h_{0}(x)=(\operatorname{tg}(x / 2))^{1-2 \mu}$. This follows easily from expansion (5.19). We have

$$
l_{0}(x)=\sum_{1}^{\infty} l_{0, k} \sin k x .
$$

As in (5.26) we find

$$
l_{0, k}=\frac{(-1)^{k} \beta_{0}^{\prime \prime}}{k^{2 \mu}}+O\left(k^{-2+2 \mu}\right)
$$

so that $(\pi-x)^{1-2 \mu} l_{0}(x)$ takes a non-zero value for $x \rightarrow \pi$.

Mathematisch Centrum Amsterdam 